The dressing was allowed to remain twenty-eight days with- the patient their decision, and if he refuses to abide by it, out interference. During this period the pulse remained $7 \overline{5}$, let him get another surgeon.

-never varying, and the temperature at no time deviated, from $98.5^{\circ}$; sleeping well, free from pain, appetite good. cheerful and contented during all this period,

June 23 , at 2 P.M., I proceeded to remove the dressing, cautiously avoiding all unnecessary movement. The wound was healthy, not one drop of pus; granulation had extended over all the replaced pieces of bone, except one small fragment, which presented a white surface. Union had progressed so far that the fibula was intact, and the tibia, at the point of fracture, was firm beyond my expectation; no swelling nor abscess presented itself; the largest piece of bone had disappeared beneath the granulating surface.

July 7, 1890, I removed the dressing for a second time. I found the wound in the soft parts filled up and firm union of the bone, the replaced pieces remaining, but still somewhat visible. The smaller piece visible at the tirst dressing much lessened, and the larger piece disappeared; no pus nor abscess present.

I may add the piece of bone next largest in size was not entirely covered by granulations.

Pulse and temperature normal, appetite excellent, sleep undisturbed. The patient's condition all that could be desired : so far not one untoward symptom has presented itself.

Dr. Kicketts, of Cincinnati, said he had read two papers in which he took the ground that in cases of doubtful fractures the surgeon had the right to make the exploratory incision. If, in a case of compound fracture, we can return the dislodged bone and have union, there is no reason why if there be two or three fragments, the surgeon should not cut down and wire them together. If we are clean there is hardly any limit to what we can do. He was glad to know that Dr. Myers did not use wire in his case, but he thought that in the majority of cases silver wire should be used to hold the pieces in position. The question of operation is a ditticalt one in railway surgery, where the medico-legal considerations are so great.

Dr. Edwin Ricketts had heard some surgeons say that the bones will not unite if the periosteum is not in place. He could not understand why this should be, if in abdominal surgery portions of the peritoneum are taken off and nothing thought of it.

Dr. North, of Michigan, said he had seen a great deal of railroad surgery, and he strongly advised against the use of the knife if it can be avoided, even if the products of ulceration flow out of the wound. Even a year afterwards an operation can be successfully performed, if necessary.

Dr. Ricketts, of Cincinnati, added that in operation on the skull, the button of bone is returned without the periosteum, and union takes place.

Dr. Griffith stated that he had used decalcified chips of bone and had bone develop from them, and he did not think the periosteum was necessary.

Dr. McCaul, of Michigan, reported a case of trephining in which he returned the button without the periosteum, and the wound is perfectly sealed to-day.

Dr. Emmett, of Iowa, thought there was no longer any question as to bone grafts growing. He had seen cases in which Senn's decalcified bone grafts were used with perfect suecess.

The chairman, Dr. Gaston, stated that his observations in this line confirmed the views to which he had just listened. He related a case of extensive suppuration along the tibia denuding two-thirds of the anterior part of the bone of its periosteum. Amputation was proposed, but it was decided to wait, and to-day the case promises complete recovery.

Dr. Emmett related a case of which he had read, in which a patient with a seriously injured foot was advised to have it amputated, but refused. Gangrene set in and the leg had ultimately to be amputated above the knee. The man sued the physician for damages, on the ground that it was the physician's duty to use his own judgment. He won the suit and the Supreme Court sustained the decision, saying that the surgeon was liable for damages, as the patient could not be supposed to know whether amputation is necessary or not.

Dr. North inquired as to the course to pursue if, in spite of every treatment, non-union continues.

Dr. Duffield, of Detroit, stated that if the Supreme Court of the State of New York decides that a patient can say that he will not have his limb amputated, and then holds the surgeon responsible for not operating, the surgeon occu-

pies a peculiar position. He had always contended that the patient should decfde. The best way is to call counsel, tell

Dr. Ricketts, of Cincinnati, replying to Dr. North, stated that mercuric chloride in large doses internally would cause union of the bone.

Dr. Myers said that he has had good results from sawing off the extremities of the bone and nailing them together. He had seen Mr. MacEwen, of Glasgow, after trephining, break up the button into small fragments and replace them. He thought it would always be better to replace any fragments removed, and recalled a case of trephining in which he did not replace the bone, and now, eight years after, there is a concave surface which is making pressure, as is shown by commencing paralysis.

Dr. Ridlon, of Chicago, speaking of non-union, referred to two cases of compound fracture in which non-union persisted in spite of all the numerous plans tried. His plan was to put the limb in position and hold it there, preventing motion laterally and longitudinally, and then constrict the limb above the point of injury by a band, producing odema about the injured part. The legs were allowed to hang down, and in both cases union was perfectly solid. Eighteen months and a year respectively elapsed between the injury and this treatment. Thomas reported forty cases so treated successfully.

Dr. Milton, of New York, related a case of non-union cured by the patient being allowed to use the leg.

Dr. Graves, of Michigan, thought that cases of non-union were really cases of delayed union. He had seen many cases in which the bones took months to unite.

Dr. Smith, of Michigan, believed that the upright position, together with getting the patient out into the fresh air, materially helped cases of non-union.

The Chairman reported a case of non-union cured by forcibly rubbing the ends of the bone together so as to excite inflammatory action.

\section{GUNSHOT WOUNDS OF THE BRAIN.}

Read in the section of Surgery and Anatomy, at the Forty-third Annual Meting of the American Merical Association, hel at Detroit, Yich, June 8, 1892 .

BY C. E. RUTH, M.D., OF KEOKUK, IOWA.

Gunshot wounds of the brain have always been considered almost of necessity fatal, and when we review the surgical history of our late war and find among hundreds of brain wounds, that not more than ten cases made partial or complete recovery, we need not be surprised to find the general concensus of medical opinion to the effect that nothing could be done in those cases but to make the patient as comfortable as possible, while the fatal result was confidently awaited.

Less than ten years ago, I have heard leading surgeons stand before medical classes and say, when a missile has entered a cavity, never explore for it. None would think of following that advice in regard to the abdomen to-day. We may not hope to accomplish,in gunshot wounds of the cerebrum, as much as has been or can be accomplished in abdominal gunshot wounds. Yet I think there is evidence to prove that cases in which missiles have entered or passed through the brain, are not necessarily so hopeless as has been believed. Many do not die immediately, and when they do not, they are entitled to as much consideration and effort to spare their lives as for other grave injuries.

All our standard authorities on surgery, which I have seen, except two, Wyeth and Treves, agree that we are not justified in attempting to follow a ball that has entered the brain. That its track can not be followed without doing more damage to the brain than the missile would do if left alone. That the finding of a ball that has entered the brain is simply a piece of surgical good fortune or accident, and not due to the operator's diagnostic or operative ability. 
The advance ground that Wyeth and Treves take seems to be based wholly on the case of Fluhrer, in Bellevue Hospital, in 1884, in which he followed the track of a ball some distance into the brain and removed a trephine disc from the opposite side of the head from the point of entrance, at a point slightly below the spot he expected the ball to have struck, and found the ball imbedded in cerebral tissue and removed it.

Cuse 1.-March the 3rd, 1891, I was called by Dr. F. L. Darrow, to see E. C., aged eighteen, who had been shot sixteen hours before by a thirty-two calibre pistol at short range. The ball entered at the outer canthus of the right eye, through the frontal process of the malar bone, and passed upward and backward into the brain. From the position of the parties concerned in the shooting, appearance and course of the wound through external soft and osseous tissue, the course of the ball through the brain was towards the opposite parietal eminence. The only symptoms of compression were slight slowing and irregularity of the pulse, which disappeared on the removal of a coagula occluding the wound and discharge of cerebral tissue and fluid blood. Temperature, pupils, motion,sensation and coördination were normal. For two weeks he remained rational, slept and ate well, pain slight. At the end of that time he began to show evidence of septic trouble with pyrexia of moderate degree, followed by anorexia and rapid failure of strength. The wound had not been allowed to close, but not being satisfied that the drainage was as complete as it should be, I carefully introduced a director four inches, and replaced it with a drainage tube. The director did not pass in the direction I had mapped out for it towards the opposite parietal eminence, but at an angle to its line of incidence of about thirty degrees. Noting no improvement in his condition, two days later I introduced a drainage tube six inches without any marked resistence. I did not know how much resistence a normal brain should offer, but was satisfied that the force I had used was so slight that I was certainly in the track of the ball. Still noting no improvement in his condition, but on the contrary rapid failure, I was satisfied that there was a pus cavity about the ball and determined to go still deeper for drainage purposes, and passed my director, and afterward drainage tube to the skull behind. The case by this time was so desperate that no time was to be lost, as he might apparently die at any time, I determined to try and find the ball. I shaved the occiput, carefully passed a gum director until it was resisted by the skull posteriorally and with a straight edge sighted the projecting end of the director vertically and horizontally, and drew a line across the occiput at right angles to the projecting portion of the straight edge in both positions. The point where the lines crossed I expected to indicate the position under which rested the point of impact of the bullet, and the tip of the director. On removing a half inch disc there was a discharge of pus to the amount of one to one and one-half ounces through a hole in the dura mater made by the impact of the bullet, and the mark of the bullet was plainly shown by a lead deposit on the inner side of the disc.

The center of impact was missed by the center of the trephine disc not more than three-sixteenths of an inch. The disc was removed at the junction of the parietal and occipital bones, one inch to the right of the median line. A drainage tube was passed entirely through the middle and posterior lobes of the brain on the right side and irrigation practiced twice daily with sterilized water and hydrogen peroxide. Appetite improved and pyrexia grew less for four or five days, during which time he was perfectly rational, but the improvement was not maintained and he died from exhaustion ten days after the operation and thirty days after the shooting occurred. The ball was procured at a dressing a couple of days after the operation. The ball being free in the abscess which formed around it made it impossible to procure it at the time of the operation without destroying normal cerebral tissue, or the removal of another trephine disc to procure more room. His condition was not such as to warrant further operative interference at that time. Unfortunately no post-mortem could be obtained.

Case 2.-May 4th, eighteen hundred and ninety-two, was called to see Fred. S., German, aged eighty, who had shot himself about thirty-six hours previously with a thirtyeight calibre pistol. The ball entered one-fourth of an inch to the right of the median line of the frontal bone and one and one-fourth inches above the supra-orbital margin. saw him. A few hours after the injury he appeared for a time quite rational and talked some. When seen by me his pulse was one hundred and eight to one hundred and twenty, of fair volume, respirations irregular and semi-comatose. Left eje normal,right palpebral tissues greatly swollen and discolored from hæmorrhagic extravasation, the eye markedly more protuberant than its fellow, and pupil insensible to light. I increased the size of the external wound and removed burnt and discolored tissue from its margin,together with a small bullet fragment. On opening the wound there was a small hæmorrhage which carried out some loose cerebral tissues. A three-sixteenths of an inch porcelaintipped probe passed readily along the bullet's track downward and backward through the frontal sinus into and through the anterior inferior portion of the first frontal convolution, through the junction of the ethmoid, frontal and body of the sphenoid bones on the right side, and lodged beneath the pharyngeal mucous membrane behind the right posterior nares, from which it was readily removed. A drainage tube was passed through the wound and allowed to remain. He survived the shooting sixty hours and was semi-comatose after the first ten hours with involuntary frecal evacuations and urinary retention. The gravity of the injury in one so old, enfeebled by insufficient food. extremely un-hygenic surroundings before and after the shooting, makes comment on the result unnecessary. There was not the slightest difficulty in following the ball through the cerebral tissue, the resistance being ample to enable me to determine that the probe was following the bullet's track.

Case 3.-In the ris Medicatrix, published at Des Moines, Iowa, February or March, 1892, Dr. Conniff reports the case of a man on whom he held a post-mortem and found a conoidal ball weighing one hundred and twenty-six grains lodged in the left posterior lobe of the cerebrum. It had passed through four or five inches of bone and soft tissue before entering the brain, and was so far spent as not to be able to reach the opposite side of the skull from this point of entrance, and probably had nearly or quite all of the grease and dirt removed before it entered the brain, and was carried twenty-nine years, the patient dying from other causes. This is, I believe, the longest period which any man has carried a ball in his brain and retained a moderate degree of physical and mental ability. Yet he complained of great pain throughout a considerable of the period, and always claimed he could feel the ball in his brain.

War histories, thus far, are of but little aid to us in determining what should be the mortality of gunshot wounds of the brain, for feeble, if any attempt, has been made to treat such wounds aseptically. In our surgical history of the Rebellion, statements are made to the effect that the wound was healthy and suppurating nicely when applied to wounds of the brain, while now there is scarcely a surgeon to be found who does not prefer to have all wounds heal without pus formation. The case of Fluhrer, as well as my cases,prove that a ball may be followed through the brain and in some cases removed. When a ball enters the brain, it may confidently be expected to pass through the cerebral mass in a straight line until it strikes bone, lodges by the resistance the cerebral tissue offers, or is deflected by the falx-cerebri or tentorium. If the missile takes a straight course throngh the cerebral tissue, all instruments, probes, directors and bullet forceps should be straight, so that the position of their tips may be accurately known, and it will be wise to have them graduated on the shaft, to enable the operator to determine the degree of penetration, and being straight, if the ball be deflected at any point, it will be shown by the probe or director persistently hugging one side of the wound of entrance, to enable it to make the curve and follow the course of the projectile, thus indicating the direction of deviation, and when that is known its extent may be determined by bending the shaft if a suitabiy tipped probe be used. 
In Treve's" Operative Surgery," just published,the statement is made that "the chief end effected by the surgeon in a large number of cases consists in providing free drainage and not in removing the foreign bodies, the presence or position of which is uncertain. It is a question which can do the more harm, a motionless bullet (which may in time become encysted), or a probe driven hither and thither through the brain substance."

He advises the use of an aluminum probe, as advocated by Fluhrer, and states that it must be allowed to pass by its own weight alone. There can be no excuse for thrusting a probe in any direction through normal brain tissue in the search for a ball. If the probe be of proper size and shape, it will give a definite and readily appreciable resistance before making a false passage. I am aware that this may be thought altogether too sweeping a statement, when the general opinion among medical men is to the effect that cerebral tissue cannot offer resistence to a probe sufficient to be appreciated by the touch, "except it be especially skilled," as one authority puts it. It would appear to be a very difficult matter to find a man specially skilled in this department. I found by careful test of many sections of brain in which post-mortem changes had not softened it,and no hardening fluid had been used upon it, that a hemisphericaly tipped probe of one-fourth inch diameter, required from two and one-half to three ounce weight to produce penetration, and one and three-fourths to two ounces to cause it to pass between the convolutions. This sized tip is sufficient to use in following any ball from thirty-two calibre upward, with a resistance to penetration that a very unskilled touch ought to appreciate, and if he cannot, he can hang on weights within safelimits, or abandon the case to some one better suited to the work,instead of trusting to his extremely uncertain touch. A three-sixteenth of an inch probe will require, under the same conditions, from one to two ounce weight to cause penetration of sulci or normal cerebral tissue, and will answer for all the small rifle balls, but both should be porcelain tipped for obvious reasons, and be carried by a small aluminum shaft, so as to give the least possible weight to the probe, and as slight lateral friction as possible to the collapsed canal, that all the resistance can be appreciated by the hand manipulating the probe, and not to be compelled to take up an appreciable part of it in resisting weight, and to have as nearly as possible all of the resistance at the probe's tip.

The external wound may give but a poor index of the course the ball takes through the brain, even though the individual shot and the one doing the shooting at the time the weapon was discharged may be known with a fair degree of accuracy. All men who have given the subject any attention, know that after a ball of any ordinary calibre passes into or through the brain there is a collapse of the walls of the track to complete filling by débris and normal cerebral tissue that has prolapsed into the track of the projectile.

Wyeth states that a gum catheter, presumably one such as is used with a stylet, a few sizes smaller than the missile, was as good a probe as could be procured. It would give more resistance to penetration than the ordinary probe. The principal objection to it is that the tip is frequently imperfect, that the shaft is the same diameter as the tip throughout, and the collapsed bullet hole produces more and more friction and consequent obstruction to the advance of the probe. It follows that before the probe advances far, the resistance may be so multiplied that it is impossible to determine whether your resistance is at the tjp of the probe or in the lateral walls. Such at least has been the experience in my hands in one experimental case, in which, for the above reasons, I made a false passage. Yet in the first operation I made on the living subject and followed the ball successfully, I used a gum catheter with a stylet to keep it straight.

If a ball be completely flattened and far spent, its course may be like a boomerang through the brain, but such balls will seldom enter the brain at all, and all others will take a straight course.

As an aid to accuracy in locating the nearest point on the scalp to the tip of a probe which had been introduced along the ball's track in the brain, I had a straight bar made ten inches long with two points three and one-half inches long projecting in the same line at right angles to the bar, one point being curved and adjustable, so that when it was placed over the head it could be adjusted to the size and shape of the head; and the bar sighted with the projecting end of the probe the same as though it were an ordinary straight edge; and with one end projecting by the side of the probe and the straight bar over the top of the head sighted with the probe, a mark may be made by the side of the opposite point which must pass over the tip of the probe, and by repeating the sighting over the skull ninety degrees from the former point and again drawing a line as before, the lines must cross each other at right angles, and the point of crossing will mark the position of the tip of the probe if the sighting be accurately done. An ordinary straight edge may be used as previously mentioned, yet this is quite an aid to additional accuracy.

If the ball strikes at right angles to the surface, or within fifteen degrees, and does not penetrate, the missile will probably lodge at the point of impact or within one-half inch of said point, and will but rarely rebound. Accepting Fluhrer's statement that a ball would rebound, I was surprised to find after firing balls into skulls, and they did not produce penetration of the opposite wall, that they remained where they struck or glanced, but did not rebound. In rare cases they re-penetrate the brain after striking the opposite bony wall; but they almost invariably do it by glancing from the point of impact, at angles to the line of incidence of more than ninety degrees. I could on no account consider that a ball rebounded, unless it re-penetrated the brain by passing back from its point of impact, at an angle to the line of incidence of less than ninety degrees.

Not finding skulls sufficiently numerous on which to experiment, I had a hemisphere made of sheet steel with every possible facility made for aiding in causing bullets to rebound, and fired twenty-two, thirty-two and forty-four calibre shots into it at all angles; and in all the varying velocities which the bullets would stand, from slight deformity to complete flattening of the balls. I had no bullet rebound that was not flattened at least one-third, and a velocity which will produce one-third flattening will nearly always produce penetration of the skull. In those which did rebound it was in varying degrees from almost the exact line of incidence to near a right an- 
gle. The distance which a ball will move from its the rongeur. I have been able to trace no evil repoint of impact after passing through the brain must sults to the use of the rongeur, yet in my experience depend upon its velocity, angle of incidence, the sur- with it I have been compelled to separate the duraface of contact, and shape of the ball. Unless the mater from the bone for some distance beyond the force of impact be great, the dura will not be cut, bone gnawed off; and should the wound suppurate, but will brobably be congested and the ball may re- it makes a point difficult to drain and increases the main between the dura mater and the brain. There liability of an unfavorable issue. A large disc can can be no objection in any such case to opening the be removed almost as quickly, easily and safely as a skull opposite to the point of entrance at the point small one, and if it be removed with as complete of secondary impact of the bullet, and establishing knowledge as is obtainable of the direction the ball drainage and removing the bullet if it be accessible. will take from the point of impact (if it moves at all), Whether the bullet be found or not, we can expect no will probably give ample room for all further operaharm to come from the trephine hole which will tive work in that case. The disc should not be reprobably prevent the formation of an abscess around placed for reasons apparent to all. Cerebral localithe bullet, and its destroying the life of the patient, zation is usually of little use in cases of this kind, as it did in my case, after the boy had shown him- except rarely when abscesses form, and then it is too self capable of recovery from the damage done to his late for operatire measures to save but a very small brain in the missile's passage: but he could not re- per cent. of cases. If the ball be small, and especover from the abscess and cerebral traumatism, and cially if it be round, has passed.through considerathe abscess could not drain through the entire cere- ble tissue before reaching the brain, so that one may bral mass along a collapsed bullet hole.

Had I removed the disc earlier, the ball would have been readily found, the track of the wound could have been drained, and the formation of an abscess around the ball, or at its point of impact, made an impossibility. After a ball has traversed the brain until it strikes the opposite bony wall, if by glancing or rebounding it is again caused to penetrate the brain, it can be expected to do so in a straight line from its point of impact, and may be followed in its secondary track to its point of iudgment, with as much ease through a trephine hole as it was originally followed from its point of entrance to where it was deflected from its course. Should it strike with considerable velocity at an angle to the horizontal of less than thirty-five degrees it may sweep around the skull in close contact with the bone for a great distance, passing probably between the dura and piamater, and leaving but little evidence to show the track it took. Occasionally balls are so far spent as to lodge in the brain at some point after gaining entrance before reaching bony resistance, and again after deflection, when it becomes necessary to remove them by some means.

I found I had no forceps at my disposal which could be operated through so small a space as the average bullet hole, without lacerating the cerebral tissue. So I had Truax \& Co., construct me a bullet forcep which could be operated through a cannula smaller than a twenty-two calibre pistol ball, and yet the jaws be opened wide enough to grasp firmly a forty-four to fifty-five calibre ball. The jaws are operated on the same principle as is used in some vertebrated or flexible csophageal forceps, but the shaft is straight and not flexible, so as to enable the surgeon to determine with certainty the position of its tip. I find that it requires considerable experience and manipulative dexterity to readily grasp and hold a missile in so soft a tissue as the brain without doing damage to the surrounding brain substance. I would not recommend anyone to attempt to grasp a bullet and extract it from the brain of the living, unless he had some experience in the work on the cadaver. I would not advise the use of a trephine less that three-fourths of an inch in diameter for removing a disc over the point of impact of a bullet, as it gives insufficient room for examination with the finger or further operative interference; unless another disc be removed, which requires considerable additional time, or the opening be enlarged by be tolerably certain that it carries in no grease and dirt, and no symptoms develop, it may be allowed to remain, otherwise it should be followed and remored if possible. In a majority of the cases pieces of bone will be broken off and carried in to the brain and lodge at various points along the bullet's track, and they should be removed when accessible, as they must of necessity act as foreign bodies, producing considerable irritation, and probably suppuration to aid in disposing of them. Re-penetration of the brain by a glance or a rebound can be expected to occur but once. The ball will pass almost invariably in a straight line from where it glanced or rebounded. There exists a necessity for trephining but once, unless it be necessary to trephine at the point of entrance, for the purpose of giving more room for elevating depressed bone.

The operative rules laid down in standard authorities regarding the enlargement if need be of the wound of entrance, removal of spicula of bone, trephinıng, etc., are too complete to justify an attempt on my part to improve on them. I have purposely omitted from this paper the tiresome details of the experiments I have made in this work, and if necessity require it, I may at some future time place them before the profession.

sUMMARY.

1. That a ball can be followed in its course through the brain.

2. Having been followed to its point of impact on the opposite side of the skull, a trephine disc should be removed, drainage be established and the ball removed if possible.

3. That the probe is best which gives the greatest resistance to penetration with least possible lateral friction on its shaft by the collapsed canal.

4. That hemispherical front, porcelain tip, and aluminum shaft answers the indications for lead detection (resistance if of proper size, weight, etc.,) required in a probe, better than anything else.

5. That one intending to follow balls through the brain should thoroughly familiarize himself with the resistance the normal brain offers to penetration by the probes he expects to use, that he may know when he is applying force within safe limits.

6. That he should frequently grasp and remove balls and pieces of bone with the forceps of his choice on the cadaver, before attempting it on the living human subject. 